\title{
Autism Spectrum Disorder: What Physicians Must Know
}

\section{Anup K Bhattacharya}

How to cite this article: Bhattacharya AK. Autism Spectrum Disorder: What Physicians Must Know. Bengal Physician Journal 2018;5(2):1-2.

\section{Source of support: Nil}

Conflict of interest: None

Autism spectrum disorder (ASD) is a complex neurodevelopmental disorder. Diagnosis is based on delay or abnormal functioning in social interactions, language, and social communication. It is often associated with symbolic or imaginative play, reduced intelligence quotient (IQ) and epilepsy. In few percentages of patients having autism-like symptoms may show relatively preserved cognitive function and language skills (Asperger's syndrome). There has been a dramatic increase in the diagnosis of ASD-a recent estimate of $\sim 1 / 150$ in comparison to $\sim 1 / 1000$ in 1950 to 1990. ${ }^{1}$ A male preponderance of ASD is found in the ratio of $4: 1$. Genetic predisposition is very high in ASD, and first-degree relatives have fifty-fold increased susceptibility in comparison to general population at-large. ASDs are genetically heterogeneous- mutations in some genes (FMR1, TSC1 and 2) are associated with mental retardation without ASDs, and alleles of certain genes like neurexin1 are associated with both schizophrenia and ASDs.

Intelligence quotient (IQ) is not considered to be a defining criterion for ASD. However, most of the children with ASD are intellectually disabled. Long-term outcome in ASD depends on IQ. Individuals with low IQ (less than 50 have a poor overall outcome.

Qualitative deficits in the following three notable characters can be found in different combinations to account for several categories of pervasive developmental disorders (PDD):

- Behavioral

- Social

- Language communication.

\section{Professor}

Department of Medicine and Incharge of Neurology, Shri Ramkrishna Institute of Medical Sciences, Malandighi, West Bengal, India

Corresponding Author: Anup K Bhattacharya, Professor, Department of Medicine and Incharge of Neurology, Shri Ramkrishna Institute of Medical Sciences, Malandighi, West Bengal, India, e-mail: anupbhattachary@yahoo.co.in

\section{Classic Autism}

Asperger's syndrome-narrow range of interest, deficient sociability, no speech delay, no mental retardation.

Pervasive developmental disorders (PDD) not otherwise specified: Autistic behavior but does not fulfill the other variants.

Heller's disintegrative disorder: Normal kids with regression in mental faculty between 2 to 10 years of age development of dementia, but no schizophrenia.

Rett's syndrome: Girls with acquired microcephaly, lack of hand use, stereotypes (hand-wringing).

Diagnostic and statistical manual of mental disorders (DSM)-IV criteria for the diagnosis of autistic behavior.

\section{Social Interaction Impairment}

- Poor eye contact, abnormal body postures with abnormal facial expression.

- Lack of social and emotional reciprocity,

- No development of peer relationship.

\section{Communication Impairment}

- Delayed development of speech,

- Even in patients with adequate speech impairment in sustain conversation.

- Stereotyped language.

\section{The Restrictive Pattern of Behavior and Activities}

- Persistent preoccupation with one object,

- Stereotyped mannerism (hand or complex body posturing).

- Specific adherence to rituals.

- Symptoms are present in early childhood but often manifest when social demands

- Increase with advancing age.

\section{Speech Defects in Autism}

Delayed development of speech, even loss of speech.

- Prosody of speech: High pitched voice, monotonous or robotic speech.

- Phonology: Word deafness

- Syntax: Agrammatism

- Semantics: Impaired comprehension, word retrieval problems, palilalia, echolalia.

- Pragmatics: Poor interpretation of voice, facial or 
bodily gestures, nonstop queries, failure to initiate or terminate sentence.

\section{Other Neurological Findings in Autism Spectrum Disorder}

Epilepsy is the commonest association in ASD around $30 \%$.

Involuntary movement in the form of stereotypes, choreoathetoid movement, motor tics, hand-flapping etc.

There may be generalized hypotonia, clumsiness of movements and toe-walking.

Limbs and vocal stereotypes, a self-injurious behavior may be there (related to increased Endorphin levels in the blood.)

\section{Neuropathology}

Increased brain volume is the most consistent finding. It is mostly due to increased white matter density. Regions mainly concerned with social functions like frontal lobe, temporal cortex, and amygdala show abnormalities in functional imaging and the same findings are seen with the orbitofrontal cortex and caudate nucleus related to stereotypes and other involuntary activities. Abnormal connectivity in the white mater along with abnormalities in mirror neurons areis the current explanation for the pathogenesis of autism.

\section{Management $^{2}$}

Special education in a home-based behavioral modification system-applied behavior analysis, social intervention therapies are still under evaluation.
Medications that are to be tried as an adjunct:

- Impulse control behavior: Mood stabilizers like divalproex sodium, carbamazepine, lithium.

- Hyperactivity and inattention: Methylphenidate, clonidine.

- Obsessive behavior: Selective serotonin reuptake inhibitor (SSRI), neuroleptics-risperidone, olanzapine, ziprasidone.

- Stereotypes: Haloperidol, risperidone, deep brain stimulation (DBS).

- Self mutilation: Fluoxetene, naloxone, clomipramine, lithium.

- Seizures: Adrenocorticotropic hormone (ACTH), divalproex.

- Psychosis: Olanzapine, risperidone, haloperidol.

The major issue remains early diagnosis and early interventions. The ultimate aim is the children get properly diagnosed and helped as per condition prevailing. To date, there is no cure for ASD. Treatment is aimed at both the short-term and long-term needs of the family and the child as a whole. The multidisciplinary approach involving primary care physicians, social workers, physicians, psychiatrists, and occupational therapists are a key to success. Parents are suited for co-therapists in the whole process.

\section{REFERENCES}

1. Nass R, Ross G. Developmental Disabilities in" Bradley's Neurology in Clinical Practice." 6th Ed. Elsevier Saunders, Philadelphia 2012. pp 1422-1443.

2. Kunju PAM. Approach to Autism Spectrum Disorder in IAP Textbook of Pediatric Neurology Ed. Anoop Verma, IAP National Publishing House, Gwalior, Jaypee Brothers Medical Publishers (p) Ltd.Chapter 25. pp 239-244. 